\title{
Screening of dry eye disease in visual display terminal workers during occupational health examinations: The Moriguchi study
}

\author{
Motoko Kawashima $^{1}$, Motoko Yamatsuj ${ }^{2}$, Norihiko Yokor ${ }^{3}$, Masaki Fukui ${ }^{1}$, \\ Yoshiyuki Ichinashi ${ }^{1}$, Hiroaki Kato ${ }^{3}$, Mitsuko Nishida ${ }^{4}$, Miki UCHINO $^{1,5}$, \\ Shigeru Kinoshita ${ }^{3}$ and Kazuo Tsubota ${ }^{1}$
}

${ }^{1}$ Department of Ophthalmology, Keio University School of Medicine, Japan, ${ }^{2}$ Yamatsuji Clinic, Japan, ${ }^{3}$ Department of Ophthalmology, Kyoto Prefectural University of Medicine, Japan, ${ }^{4}$ Health Care Center, Panasonic Health Insurance Organization, Panasonic Corporation, Japan and ${ }^{5}$ Harvard School of Public Health, USA

\begin{abstract}
Screening of dry eye disease in visual display terminal workers during occupational health examinations: The Moriguchi study: Motoko KaWASHIMA, et al. Department of Ophthalmology, Keio University School of Medicine-Purpose: The aim of this study was to estimate the prevalence of dry eye disease (DED) in Japanese visual display terminal (VDT) workers and identify simple methods for screening DED during occupational health examinations. Methods: This study involved 369 (331 men and 38 women; mean age, 44.4 [8.8] years) workers engaged in PC development with approximately 6 hours of VDT use daily. The participants completed questionnaires on dry eye and physical symptoms affecting QoL, and their ability to keep their eyes open for 10 seconds was tested for DED screening. They also underwent assessment of conjunctival and corneal vital staining with lissamine green and fluorescein, measurement of tear film breakup time, and Schirmer's test for DED diagnosis. Sensitivity, specificity, and positive predictive value of the screening methods were assessed. Results: The DED group included 218 (definite, 14; probable, 204) participants. They had markedly high frequencies of 11 dry eye symptoms, especially ocular fatigue (OR, $17.1 ; 95 \% \mathrm{Cl}$, 9.9 to 29.7$)$ and dry sensation (OR, $8.2 ; 95 \% \mathrm{Cl}, 3.6$ to 18.4), and were more often unable to keep their eyes open for $10 \mathrm{~s}$. With either ocular fatigue or dry sensation as the cutoff criterion, the sensitivity, specificity, and positive predictive value were $89.9,66.9$, and $79.7 \%$, respectively. Conclusions: Approximately $60 \%$ of the VDT workers were diagnosed with DED on the basis of diverse eye symptoms affecting their ability to work. The dry eye symptom questionnaire and 10 -s eye-opening
\end{abstract}

Received Oct 26, 2014; Accepted Jan 10, 2015

Published online in J-STAGE Mar 6, 2015

Correspondence to: $M$. Kawashima, Department of Ophthalmology,

Keio University School of Medicine, 35 Shinanomachi, Shinjuku-ku,

Tokyo 160-8582, Japan (e-mail: motoko-k@a3.keio.jp) test are simple, noninvasive, and inexpensive methods for screening DED during occupational health examinations.

(J Occup Health 2015; 57: 253-258)

Key words: Dry eye disease, Occupational health examination, Ophthalmologists, Quality of life, Screening, Visual display terminal

Dry eye disease (DED) is a multifactorial condition causing ocular discomfort, visual disturbance, and tear film instability with potential damage to the ocular surface $^{1)}$. Occupational changes such as increased VDT use have raised its prevalence among office workers in Japan. For example, $10.1 \%$ of the men and $21.5 \%$ of the women in an epidemiological study of Japanese office workers using VDTs were clinically diagnosed with DED; severe symptoms were observed in $26.9 \%$ and $48.0 \%$ of the male and female subjects, respectively ${ }^{2}$.

VDT use is a remarkable risk factor for dry eye symptoms ${ }^{2,3)}$. The daily duration of VDT use is linearly related to dry eye symptoms ${ }^{4}$. Prolonged use is associated with symptoms such as dryness, ocular fatigue, and visual difficulty, which are reported more often than musculoskeletal pain and mental stress ${ }^{4}$. However, current occupational health examinations for VDT users cover only some dry eye symptoms, and DED screening is not routinely performed ${ }^{5)}$. Further, few reports of occupational health examinations focusing on DED have been published ${ }^{6,7)}$.

The aim of this study was to estimate the prevalence of DED among Japanese VDT workers and identify simple methods for screening DED during occupational health examinations. 


\section{Methods}

Study population

This study was performed following a request by occupational physicians at AVC Networks Company, Panasonic Corporation, Osaka, Japan. The Personnel Affairs Department of AVC Networks Company sent e-mails regarding examinations for DED to all employees involved in development, design, sale, and service of personal computers and tablets. We enrolled employees who had approximately 6 hours of daily VDT use, were willing to participate, and gave written consent after receiving an explanation on personal information protection and the study's purpose.

This study followed the tenets of the amended Declaration of Helsinki and was supervised by the Dry Eye Society, Tokyo, Japan. The protocol was approved prospectively by the Institutional Review Board of Ryogoku Eye Clinic, Tokyo, Japan, and the Safety Hygiene Committee of AVC Networks Company.

\section{Questionnaire}

We administered a web-based questionnaire to obtain information on age, gender, VDT use (duration, operational type, and monitor position), dry eye symptoms, and symptoms affecting QoL. The questionnaire covered 12 dry eye symptoms: ocular fatigue, pain, discharge, foreign body sensation, excess tearing, blurred vision, itching, heavy sensation, redness, uncomfortable sensation, dry sensation, and sensitivity to bright light ${ }^{8}$. The frequency of each item was evaluated as "never" (1 point), "sometimes" ( 2 points $)$, "often" (3 points), and "constantly" (4 points), and the mean of all the points was defined as the dry eye symptom score. Participants who answered "constantly" or "often" for at least 1 item were considered symptomatic. The QoL items included questions on whether or not participants had ever had trouble at work due to eye symptoms and physical symptoms such as fatigue and pain in various body parts (head, neck, shoulder, arm, hand, finger, back, waist, and foot). We also tested whether participants could keep their eyes open for 10 seconds.

\section{Clinical evaluation}

Five ophthalmologists from the Dry Eye Society performed the ophthalmic examinations at the Health Care Institute of AVC Networks Company in Moriguchi, Osaka, Japan. The examinations included assessment of conjunctival and corneal vital staining with lissamine green and fluorescein, measurement of tear film breakup time (TBUT), and Schirmer's test, which were perfomed as reported previously ${ }^{6}$.

\section{DED diagnosis}

DED was diagnosed on the basis of the criteria defined by the Dry Eye Society in 20069). These criteria include presence of (1) dry eye symptoms; (2) qualitative or quantitative abnormalities of the tear film in one or both eyes (Schirmer's test results of $\leq 5 \mathrm{~mm}$ or TBUT of $\leq 5$ seconds); and (3) conjunctivocorneal epithelial damage (total staining score of $\geq$ 3 out of 9 points). Participants meeting all the criteria were considered to have definite DED, and those meeting 2 criteria in one or both eyes had probable DED; the presence of 1 or absence of any criterion indicated no DED. Participants with definite and probable DED were classified into the DED group.

\section{Statistical analysis}

Discrete variables were compared using the Student's t-test, and categorical variables were compared using Fisher's exact test or the chi-square test. $p<0.05$ was considered significant. SAS 9.2 for Windows (SAS Institute, Inc., Cary, NC, USA) was used for the analyses.

\section{Results}

Of 414 employees approached, 369 (89.4\%) completed the ophthalmic examinations and questionnaire. Their mean (SD) age was $44.4(8.8)$ years; and $331(89.7 \%)$ were men, and $38(10.3 \%)$ were women. The proportions of individuals with definite, probable, and non-DED diagnoses were 3.8\% (14), 55.3\% (204), and $40.9 \%$ (151), respectively (Table 1). No significant age difference was noted according to diagnosis.

Duration of VDT use was significantly longer in the DED group than in the non-DED group ( $p=0.015$; Table 2). The prevalence of DED was not significantly different among the operational types, and 98\% of the participants placed the monitors at or below the horizontal plane of the eye glance level. Significantly fewer participants found 10-s eye opening impossible in the non-DED group $(p=0.032)$. The sensitivity, specificity, and positive predictive value of this screening test were $19.7,88.7$, and $71.7 \%$, respectively. Over $20 \%$ of the DED group had trouble at work due to ocular fatigue or strain, which was significantly higher than proportion in the non-DED group $(p=0.000)$. This group also had a higher proportion of other physical symptoms $(p<0.05)$.

Of the 12 dry eye symptoms, all except excess tearing were significantly more frequent in the DED group (Table 2). The dry eye symptom score was significantly higher in the DED group than in the non-DED group (3.6 [2.6] vs. 1.4 [2.5]; $p=0.000$, Student's $t$-test). Assuming 1 positive symptom as the cutoff criterion, the sensitivity and specificity were 98.2 and $62.9 \%$, respectively. With 2 positive 
Table 1. Characteristics of the study population

\begin{tabular}{llllll}
\hline \multirow{2}{*}{ Variable } & & \multicolumn{3}{c}{ Diagnosis } & \\
\cline { 3 - 5 } & & $\begin{array}{l}\text { Definite DED } \\
\mathrm{n}(\%)\end{array}$ & $\begin{array}{l}\text { Probable DED } \\
\mathrm{n}(\%)\end{array}$ & $\begin{array}{l}\text { Non-DED } \\
\mathrm{n}(\%)\end{array}$ & $p$-value \\
\hline \multirow{2}{*}{$\begin{array}{l}\text { Age } \\
\text { range }\end{array}$} & $20-24$ & $0(0.00)$ & $0(0.00)$ & $1(100.00)$ & $0.895^{1)}$ \\
& $25-29$ & $1(3.70)$ & $17(62.96)$ & $9(33.33)$ & \\
& $30-34$ & $1(3.33)$ & $16(53.33)$ & $13(43.33)$ & \\
& $35-39$ & $1(2.86)$ & $23(65.71)$ & $11(31.43)$ & \\
& $45-44$ & $2(3.45)$ & $29(50.00)$ & $27(46.55)$ & \\
\hline Gender & $50-54$ & $6(5.94)$ & $56(55.45)$ & $39(38.61)$ & \\
& $55-59$ & $1(3.03)$ & $15(45.45)$ & $17(51.52)$ & \\
& Men & $11(3.3)$ & $175(52.9)$ & $145(43.8)$ & $0.003^{1)}$ \\
\hline
\end{tabular}

${ }^{1)}$ Fisher's exact test. DED, dry eye disease.

symptoms as the cutoff criterion, the respective diagnostic values were 77.5 and $72.2 \%$. Figure 1 shows the ROC curve for sensitivity and specificity based on dry eye symptom score. The area under the curve (AUC) was 0.807. Comparison of the ORs for the dry eye symptoms between the DED and non-DED groups showed that ocular fatigue and dry sensation had the highest ORs (Table 3). Considering the presence of either ocular fatigue or dry sensation as the cutoff criterion, the sensitivity, specificity, and positive predictive value were $89.9,66.9$, and $79.7 \%$, respectively.

\section{Discussion}

In this study, approximately $60 \%$ of the VDT workers were diagnosed with $\mathrm{DED}^{6}$. This prevalence is similar to the reported prevalence of $60.2 \%$ in men and $76.5 \%$ in women based on clinical evaluation of office workers using VDTs ${ }^{6}$. Gender and age are risk factors for $\mathrm{DED}^{10)}$. Despite the small proportion of women, the prevalence of DED was higher among female participants in our study. Age, however, was not associated with DED prevalence. These results suggest that risk factors such as duration of VDT use and gender may have greater impact than age among young and middle-aged VDT workers.

Although previous studies showed no correlation between symptoms and clinical findings ${ }^{11-13)}$, dry eye symptoms are the most important criteria in screening for $\mathrm{DED}^{14-16)}$. We applied a widely used Japanese questionnaire on $\mathrm{DED}^{8}$ : 11 items except excess tearing were useful for DED screening. The McMonnies Dry Eye Questionnaire is widely used as a screening instrument for DED subjects, and has significantly higher sensitivity and specificity than those found

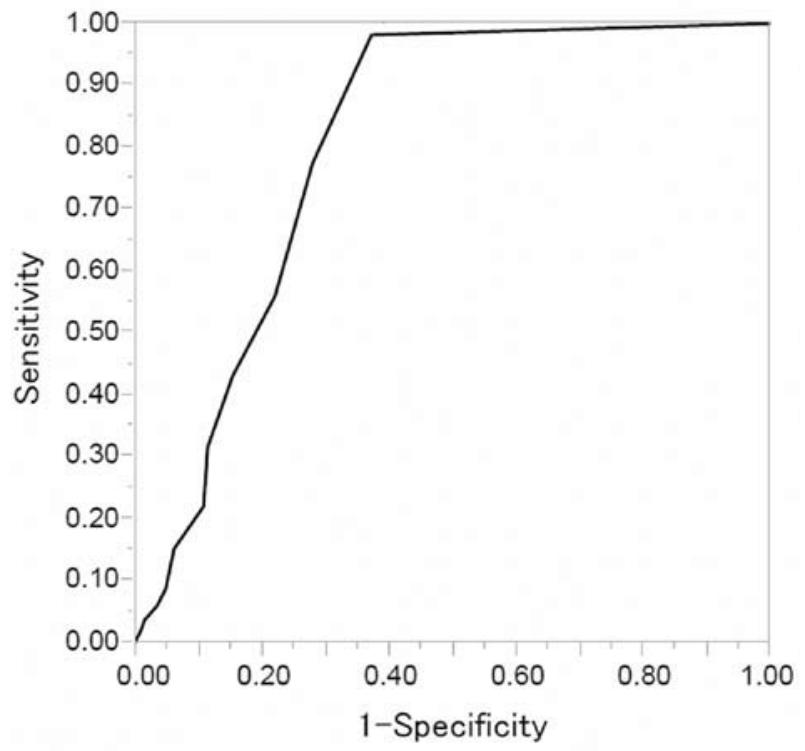

Fig. 1. ROC curve for diagnosis of dry eye disease based on dry eye symptoms.

in our study ${ }^{17}$. It is very useful in detecting the severe types of DED like the aqueous deficient type. However, it is unclear whether it can, with high sensitivity and specificity, discriminate cases of DED from non-DED in an office-working population using VDTs because this population generally has a short $\mathrm{BUT}^{6}$. Our findings suggest that higher sensitivity than specificity is necessary to avoid misclassification during occupational health examinations, which usually have lower detection rates of DED than examinations by ophthalmologists. VDT workers with 1 positive symptom should be advised to undergo ophthalmic 
Table 2. Comparison of the study groups

\begin{tabular}{|c|c|c|c|c|}
\hline \multicolumn{2}{|l|}{ Variable } & $\begin{array}{l}\text { DED group } \\
(\mathrm{n}=218)\end{array}$ & $\begin{array}{l}\text { Non-DED group } \\
\quad(\mathrm{n}=151)\end{array}$ & $p$-value \\
\hline \multicolumn{2}{|c|}{ Duration of visual display terminal (VDT) use (h) } & $6.5 \pm 2.0$ & $6.0 \pm 2.0$ & $0.015^{1)}$ \\
\hline \multirow{5}{*}{$\begin{array}{c}\text { Operational type } \\
\mathrm{n}(\%)\end{array}$} & Simple input & $11(5.3)$ & $13(9.2)$ & $0.088^{2)}$ \\
\hline & Binding & $2(1.0)$ & $1(0.7)$ & \\
\hline & Interactive & $131(62.7)$ & $69(48.9)$ & \\
\hline & Technical & $64(30.6)$ & $58(41.1)$ & \\
\hline & Monitoring & $1(0.5)$ & $0(0)$ & \\
\hline \multicolumn{2}{|c|}{ Number of missing data } & 9 & 10 & \\
\hline \multirow{3}{*}{$\begin{array}{c}\text { Monitor position } \\
\mathrm{n}(\%)\end{array}$} & Eye level & $35(17.6)$ & $18(13.9)$ & $0.438^{2)}$ \\
\hline & Below eye level & $158(79.4)$ & $110(84.6)$ & \\
\hline & Above eye level & $6(3.0)$ & $2(1.5)$ & \\
\hline \multicolumn{2}{|c|}{ Number of missing data } & 19 & 21 & \\
\hline \multirow{12}{*}{$\begin{array}{l}\text { Eye symptoms } \\
\mathrm{n}(\%)\end{array}$} & Ocular fatigue & $195(89.5)$ & $50(33.1)$ & $0.000^{3)}$ \\
\hline & Pain & $35(16.1)$ & $9(6.0)$ & $0.003^{3)}$ \\
\hline & Discharge & $56(25.7)$ & $18(11.9)$ & $0.001^{3)}$ \\
\hline & Foreign body sensation & $52(23.9)$ & $13(8.6)$ & $0.000^{3)}$ \\
\hline & Excess tearing & $40(18.4)$ & $18(11.9)$ & $0.110^{3)}$ \\
\hline & Blurred vision & $88(40.4)$ & $23(15.2)$ & $0.000^{3)}$ \\
\hline & Itching & $64(29.4)$ & $16(10.6)$ & $0.000^{3)}$ \\
\hline & Heavy sensation & $51(23.4)$ & $13(8.6)$ & $0.000^{3)}$ \\
\hline & Redness & $45(20.6)$ & $16(0.6)$ & $0.011^{3)}$ \\
\hline & Uncomfortable sensation & $53(24.3)$ & $13(8.6)$ & $0.000^{3)}$ \\
\hline & Dry sensation & $62(28.4)$ & $7(4.6)$ & $0.000^{3)}$ \\
\hline & Sensitivity to bright light & $53(24.3)$ & $15(9.9)$ & $0.000^{3)}$ \\
\hline \multirow{2}{*}{$\begin{array}{c}\text { 10-s eye opening } \\
\mathrm{n}(\%)\end{array}$} & Possible & $175(80.3)$ & $134(88.7)$ & $0.032^{3)}$ \\
\hline & Impossible & $43(19.7)$ & $17(11.3)$ & \\
\hline \multirow{2}{*}{$\begin{array}{c}\text { Trouble at work } \\
\mathrm{n}(\%)\end{array}$} & Yes & $50(22.9)$ & $11(7.3)$ & $0.000^{3)}$ \\
\hline & No & $168(77.9)$ & $140(92.7)$ & \\
\hline \multirow{6}{*}{$\begin{array}{l}\text { Other physical } \\
\text { symptoms } \\
\mathrm{n}(\%)\end{array}$} & Headache & $84(38.5)$ & $36(23.8)$ & $0.003^{3)}$ \\
\hline & $\begin{array}{l}\text { Neck and shoulder pain/ } \\
\text { fatigue }\end{array}$ & $174(79.8)$ & $88(58.3)$ & $0.000^{3)}$ \\
\hline & $\begin{array}{l}\text { Upper extremity pain/ } \\
\text { fatigue }\end{array}$ & $53(24.3)$ & $23(15.2)$ & $0.037^{3)}$ \\
\hline & Back pain/fatigue & $67(30.7)$ & $35(23.2)$ & $0.124^{3)}$ \\
\hline & Lower back pain/fatigue & $100(45.9)$ & $53(35.1)$ & $0.042^{3)}$ \\
\hline & Foot pain/fatigue & $58(26.2)$ & $160(73.4)$ & $0.000^{3)}$ \\
\hline
\end{tabular}

${ }^{1)}$ Student's $t$-test, ${ }^{2)}$ Chi-square test, and ${ }^{3)}$ Fisher's exact test. DED, dry eye disease.

examinations. The probability of DED is especially greater in those reporting ocular fatigue or dry sensation.

Spontaneous blinking rate tends to increase in patients with DED because of tear film instability ${ }^{18)}$. On the other hand, during VDT use, the spontaneous blinking rates of both normal and DED-affected eyes decreases when compared with those at rest ${ }^{19)}$. In our study, the high positive predictive value indicated that VDT workers who cannot keep their eyes open for 10 seconds have an increased probability of DED. However, the test should be used in combination with dry eye symptomatology because of its low sensitivity.

In VDT work, overall ergonomics, including monitor position, are important when attempting to decrease body fatigue and musculoskeletal pain ${ }^{20,21)}$. 
Table 3. Odds ratios of eye symptoms

\begin{tabular}{lcc}
\hline Symptom & OR & $95 \%$ CI \\
\hline Ocular fatigue & 17.1 & 9.9 to 29.7 \\
Dry sensation & 8.2 & 3.6 to 18.4 \\
Blurred vision & 3.8 & 2.2 to 6.3 \\
Itching & 3.5 & 1.9 to 6.4 \\
Uncomfortable sensation & 3.4 & 1.8 to 6.5 \\
Foreign body sensation & 3.3 & 1.7 to 6.4 \\
Heavy sensation & 3.2 & 1.7 to 6.2 \\
Pain & 3.0 & 1.4 to 6.5 \\
Sensitivity to bright light & 2.9 & 1.6 to 5.4 \\
Discharge & 2.6 & 1.4 to 4.6 \\
Redness & 2.2 & 1.2 to 4.1 \\
Excess tearing & 1.7 & 0.9 to 3.0 \\
\hline
\end{tabular}

OR, odds ratio; $\mathrm{CI}$, confidence interval.

In this study, most of the participants placed their monitors at or below eye level. Although operational type was not significantly different, participants with DED had a longer duration of VDT use. A limitation of this method was that the duration of VDT use was calculated based on the questionnaire, and was not based on an actual measured value. The results, however, concur with evidence that ocular symptoms tend to occur independent of the type of computer work $^{22)}$. Importantly, duration of computer work is directly correlated with symptoms: longer durations tend to cause long-lasting complaints well after VDT work is completed ${ }^{23)}$.

Tear film instability and corneal epithelial disorder cause visual disturbance because of irregularity of the optical zone on the ocular surface ${ }^{24-26)}$. In addition to visual disturbance, various symptoms of DED affect concentration while working. Uchino et al. reported that DED status is associated with lower work productivity and impaired work performance in relatively young VDT users ${ }^{27)}$. Productivity is also significantly lower among those with DED: the annual cost of work productivity loss is estimated to be USD 741 per person ${ }^{28)}$. For early identification and treatment of DED and its complications, as well as concomitant diseases, regular visits to an ophthalmologist should be promoted.

Taken together, the results highlight the necessity of applying noninvasive, simple, and inexpensive screening methods, such as the dry eye symptom questionnaire and 10-s eye-opening test, during occupational health examinations for VDT workers. Ophthalmologists should raise awareness of DED and work cooperatively with industrial doctors for promoting DED management in VDT users.
Acknowledgments: This study was a collaborative investigation of the Dry Eye Society, Tokyo, Santen Pharmaceutical Co., Ltd., Osaka, and AVC Networks Company, Panasonic Corporation, Osaka, Japan. AVC Networks Company provided the research facilities, and Santen Pharmaceutical Co., Ltd. assisted in the transport of equipment, data analysis, and data management. We thank Dr. Murat Dogru for his valuable advice and constructive suggestions.

\section{References}

1) DEWS Report. The definition and classification of dry eye disease: report of the definition and classification subcommittee of the international dry eye workshop. Ocul Surf 2007; 5: 75-92.

2) Uchino $M$, Schaumberg DA, Dogru $M$, et al. Prevalence of dry eye disease among Japanese visual display terminal users. Ophthalmology 2008; 115 : 1982-8.

3) Miljanović B, Dana R, Sullivan DA, Schaumberg DA. Impact of dry eye syndrome on vision-related quality of life. Am J Ophthalmol 2007; 143: 409-15.

4) Nakazawa T, Okubo Y, Suwazono $Y$, et al. Association between duration of daily VDT use and subjective symptoms. Am J Ind Med 2002; 42: 421-6.

5) Ministry of Health, Labour \& Welfare. Guidelines for VDT work in labor hygiene [in Japanese]. Tokyo: Kouseiroudoushou Kihatsu; 2002.

6) Uchino M, Yokoi N, Uchino Y, et al. Prevalence of dry eye disease and its risk factors in visual display terminal users: the Osaka study. Am J Ophthalmol 2013; 156: 759-66.

7) Uchino M, Uchino Y, Dogru M, et al. Dry eye disease and work productivity loss in visual display users: the Osaka study. Am J Ophthalmol. 2014; 157: 294-300

8) Toda I, Fujishima H, Tsubota K. Ocular fatigue is the major symptom of dry eye. Acta Ophthalmol (Copenh) 1993; 71: 347-52.

9) Shimazaki J, Tsubota K, Kinoshita S, et al. Definition and diagnosis of dry eye 2006. Atarashii Ganka 2007; 24: 181-4 (in Japanese).

10) DEWS report. The epidemiology of dry eye disease: report of the epidemiology subcommittee of the international dry eye workshop. Ocul Surf 2007; 5: 93-107.

11) Nichols KK, Nichols JJ, Mitchell GL. The lack of association between signs and symptoms in patients with dry eye disease. Cornea 2004; 23: 762-70.

12) Begley CG, Chalmers RL, Abetz L, et al. The relationship between habitual patient-reported symptoms and clinical signs among patients with dry eye of varying severity. Invest Ophthalmol Vis Sci 2003; 44: 4753-61.

13) Chalmers RL, Begley CG, Edrington T, et al. The agreement between self-assessment and clinician assessment of dry eye severity. Cornea 2005; 24 : 804-10. 
14) Schiffman RM, Christianson MD, Jacobsen G, Hirsch JD, Reis BL. Reliability and validity of the ocular surface disease Index. Arch Ophthalmol 2000; 118: 615-21.

15) McMonnies C, Ho A. Marginal dry eye diagnosis. In Holly F (ed.) The preocular tear film in health, disease and contact lens wear. Lubbock (TX): Dry Eye Institute; 1986. p.32-8.

16) Sakane Y, Yamaguchi $M$, Yokoi N, et al. Development and validation of the dry eye-related quality-of-life score questionnaire. JAMA Ophthalmol 2013; 131: 1331-8.

17) Gothwal VK, Pesudovs K, Wright TA, et al. McMonnies questionnaire: enhancing screening for dry eye syndromes with rasch analysis. Invest Ophthalmol Vis Sci 2010; 51: 1401-7.

18) Nakamori $K$, Odawara $M$, Nakajima $T$, Mizutani $\mathrm{T}$, Tsubota K. Blinking is controlled primarily by ocular surface conditions. Am J Ophthalmol 1997; 124: 24-30.

19) Schlote T, Kadner G, Freudenthaler N. Marked reduction and distinct patterns of eye blinking in patients with moderately dry eyes during video display terminal use. Graefes Arch Clin Exp Ophthalmol 2004; 242: 306-12.

20) Tsubota K, Nakamori K. Dry eye and video display terminals. N Engl J Med 1993; 328: 584.
21) Taptagaporn S, Sotoyama M, Saito S, Suzuki T, Saito S. Visual comfort in VDT workstation design. J Hum Ergol (Tokyo) 1995; 24: 84-8.

22) Scullica L, Rechichi C. Is the type of VDT work important in asthenopia? Eur J Ophthalmol 1993; 3: 37-41.

23) Gur S, Ron S. Does work with visual display units impair visual activities after work? Doc Ophthalmol 1992; 79: 253-9.

24) Koh S, Maeda N, Hirohara Y, et al. Serial measurements of higher-order aberrations after blinking in patients with dry eye. Invest Ophthalmol Vis Sci 2008; 49: 133-8.

25) Goto E, Yagi Y, Matsumoto Y, Tsubota K. Impaired functional visual acuity of dry eye patients. Am J Ophthalmol 2002; 133: 181-6.

26) Kaido M, Matsumoto $Y$, Shigeno $Y$, Ishida R, Dogru M, Tsubota K. Corneal fluorescein staining correlates with visual function in dry eye patients. Invest Ophthalmol Vis Sci 2011; 52: 9516-22.

27) Uchino $M$, Uchino $Y$, Dogru $M$, et al. Dry eye disease and work productivity loss in visual display users: the Osaka study. Am J Ophthalmol 2014; 157: 294-300.

28) Yamada M, Mizuno Y, Shigeyasu C. Impact of dry eye on work productivity. Clinicoecon Outcomes Res 2012; 4: 307-12. 